\title{
RADIATION-STIMULATED CHANGES IN THE CHARACTERISTICS OF SURFACE-BARRIER AL-SI-BI STRUCTURES WITH DIFFERENT CONCENTRATIONS OF DISLOCATIONS AT THE CRYSTAL SURFACE
}

\author{
Bohdan PAVLYK*, Markijan KUSHLYK*, Dmytro SLOBODZYAN*, Igor MATVIJISHYN*, Roman LYS*, Marek JALBRZYKOWSKI* \\ *Faculty of Electronics, Lviv Ivan Franko National University, Drahomanova Street 50, 79005 Lviv, Ukraine \\ **Bialystok University of Technology, Faculty of Mechanical Engineering, ul. Wiejska 45C, 15-351 Bialystok, Poland \\ pavlyk@electronics.Inu.edu.ua, kushlykmarik@gmail.com, slobodzyan d@ukr.net, \\ matviijshyn@ukr.net, lys_r@ukr.net, m.jalbrzykowski@pb.edu.pl
}

received 6 June 2016, revised 19 March 2018, accepted 22 March 2018

\begin{abstract}
We report the results of studies for the radiation-stimulated changes in electro-physical characteristics of surface-barrier $\mathrm{Al}-\mathrm{Si}-\mathrm{Bi}$ structures based on $\mathrm{p}$-Si. We demonstrate that the X-ray irradiation is accompanied by different processes which depend on the density of the dislocations in the original silicon crystals. A usual evolution of the existing structural defects and their radiationstimulated ordering dominate when the concentration remains low enough. Increase in the concentration causes the increasing role of generation of additional radiation defects. Modelling of the underlying physical processes has testified that the near-contact Si layers are strained. They act as getters for the structural defects and impurities.
\end{abstract}

Key words: Silicon, Dislocations, Surface-Barrier Structures, Surface Layers, Radiation-Stimulated Reconstruction of Defects

\section{INTRODUCTION}

Fabrication of reliable electronic systems with well-predicted characteristics, which are able to work in extreme conditions (e.g., in a wide temperature range, under the influence of irradiation, atmosphere or magnetic fields), is one of the urgent problems of modern electronics engineering. The external factors induce a number of changes in electro-physical and optical characteristics of crystals such as silicon and corresponding devices made on their basis.

In the recent years there have appeared a significant number of publications devoted to the regularities of changes occurring to the parameters and characteristics of surface-barrier structures (SBSs) made of silicon, as well as to diode structures subjected to the magnetic and irradiation fields (Borkovska et al., 1984; Mahkamov et al., 1999; Nikolaev et al., 2003; Marchenko et al., 2010; Kalinina et al., 2010; Dolgolenko et al., 2006; Makara et al., 2008; Skvortsov et al., 2009; Mudriy et al., 2010). It is now well established (Borkovska et al., 1984; Mahkamov et al., 1999; Nikolaev et al., 2003; Marchenko et al., 2010; Kalinina et al., 2010; Dolgolenko et al., 2006) that the irradiation stimulates evolution of defect structure of crystals and so can lead to either deterioration or improvement of many characteristics of the silicon structures. Though being somewhat unusual, the latter effect really exists and is known as an 'effect of small doses'.

There is fewer works devoted to combined influence of the mentioned fields on the processes of defects evolution in silicon, as well as to the corresponding stimulated changes in the parameters of structures on its basis (Mudriy et al., 2010). A path from microelectronics to nanoelectronics makes the studies in this field more urgent, since they can enable predicting and governing the changes occurring in the parameters of many devices of the modern electronics.

The aim of this work is to study the influence of concentration of surface dislocations on the efficiency of radiation-stimulated reconstruction of defects located in the near-surface layer of the SBSs made of $\mathrm{p}-\mathrm{Si}$.

\section{EXPERIMENTAL TECHNIQUES}

In our experiments we used $p$-Si samples with the specific resistance of $\rho_{1}=24 \mathrm{Ohm} \cdot \mathrm{cm}$, which had been grown with the Czochralski technique. Contacts at the crystal surfaces were made with the aid of a Vacuum Universal Post device VUP-5 under the pressure of $10^{-5} \mathrm{~Pa}$. A nitric-trap technique was used according to standard procedures described earlier by Pavlyk et al. (2012). A $240-250 \mathrm{~nm}$ thick aluminum film was coated, using thermal vacuum sputtering, on the (111) silicon surface, which had been preliminarily polished chemically and degassed in a vacuum (the pressure $10^{-5} \mathrm{~Pa}$ ) at $320^{\circ} \mathrm{C}$. The corresponding coating rate was equal to $15 \mathrm{~nm} / \mathrm{s}$. After that the samples were subjected to a necessary thermal treatment and cooled down to the room temperature. The other contact was a $90 \mathrm{~nm}$ thick bismuth film. It was coated with the same rate. Considering a technological peculiarity of the evaporation process consisting in $\mathrm{Bi}$ contacts being deteriorated, the samples were not treated thermally in the latter case. All the procedures involved in the formation of our SBSs were performed in a single production cycle.

For the microscopic studies of defect state of our crystals covered by the metal films, we etched the samples chemically in the two following stages: at the first one the metal film was etched using the hydrochloric acid and, at the second, the samples were 
subjected to standard etching with a selective etchant. To determine the concentration of etching pits, we used an light microscopy based upon a microscope LUMAM I-3 and an atomic force microscopy facilitated by a Solver P-4 device.

The samples were irradiated by $\mathrm{X}$-ray beams, using an X-ray equipment URS.1.0 (the Mo anode, $U=45 \mathrm{kV}$, and $I=10 \mathrm{~mA}$ ). Finally, the volt-ampere (VAC) and the high-frequency $(125 \mathrm{kHz})$ capacity-voltage (CVC) characteristics of our Al-Si-Bi SBSs were measured with a capacitive modulation spectrometer URAN-1. The data collected in this manner were processed using a personal computer. To provide different dislocation concentrations on the (111) surface of each experimental sample, we carried out controlled plastic straining of the silicon crystals at the temperature of $1000 \mathrm{~K}$.

\section{EXPERIMENTAL RESULTS AND DISCUSSION}

In order to confirm experimentally the availability of defects in the near-surface layer of our silicon crystals and determine their compositions qualitatively and quantitatively (the types of the defects, their energy levels, etc.), we measured both the VAC and the high-frequency CVC. Fig. 1a displays the VACs obtained for the SBS sample having the barrier contacts located in the areas with the concentrations of dislocations $10^{2}, 10^{4}$ and $10^{5} \mathrm{~cm}^{-2}$. As seen from Fig. $1 b$, introduction of dislocations into the $p-S i$ crystals results in decreasing direct current flowing through the SBS under direct voltage. This is mainly caused by the effect of gettering of the majority charge carriers at the dislocations.

According to a standard and well-known theory, the following relation holds true for the total current $I_{p n}$ through the $p$-n-junction:

$I_{p n}=I_{s}\left(e^{-\frac{e U}{k T}}-1\right)+I_{R}\left(e^{-\frac{e U}{2 k T}}-1\right)$.

Here $I_{S}=\frac{e D S P}{L \cdot t h\left(\frac{\omega}{L}\right)}$ is the diffusion current and $I_{R}=\frac{\frac{k T}{e} P_{n 0} D \omega}{\left(\Delta \varphi_{0}-U_{p n}\right) L^{2}}$ the recombination current, e the electron charge, $U$ the electric voltage, $k$ Boltzmann constant, and $T$ the absolute temperature. In the formulas defining the $I_{S}$ and $I_{R}$ parameters, $S$ denotes the $p$-njunction area, $D$ the diffusion coefficient, $P_{n 0}$ the concentration of impurities injected into the base, $L$ the diffusion path length, $\Delta \varphi_{0}$ the potential barrier, $\omega$ the thickness of the base, and $U_{p n}$ the voltage applied to the $p$-n-junction.

The total reverse current can be presented as a sum of the diffusion current in the neutral area and the current thermally generated inside the barrier region.

The concentration of the intrinsic charge carriers in the crystals under test is small, so that the contribution of the diffusion component is minimal and the reverse current is described well by the Shockley law. An increase in the concentration of minority carriers takes place in the course of uniaxial straining of the crystal. This factor explains the increased reverse current (see Fig. 1b).

Fig. 2a depicts the CVC for the SBSs with different concentrations of dislocations at their surfaces. It is apparent that increasing dislocation concentration decreases the capacity both near its maximum and in the region of positive bias voltages. This means that the generation-recombination processes in the space-charge region cause a decrease in the positive charge located inside the insulating substrate. In other words, the contribution of charge carriers to the capacity of the space-charge region decreases due to gettering of charge carriers at the dislocations. a)

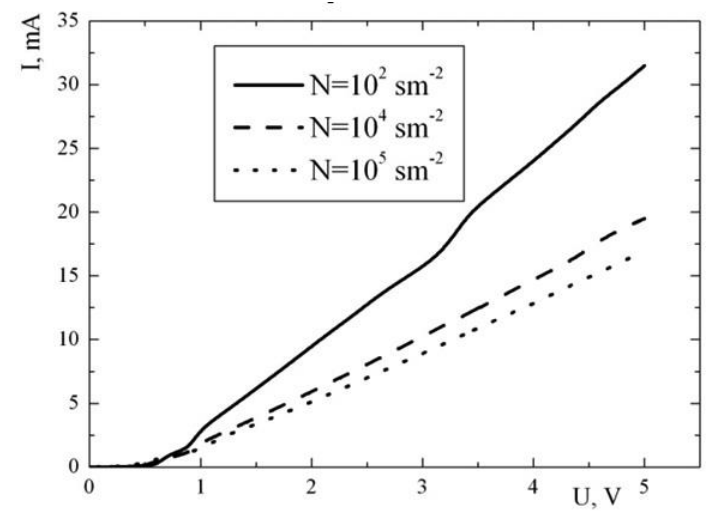

b)

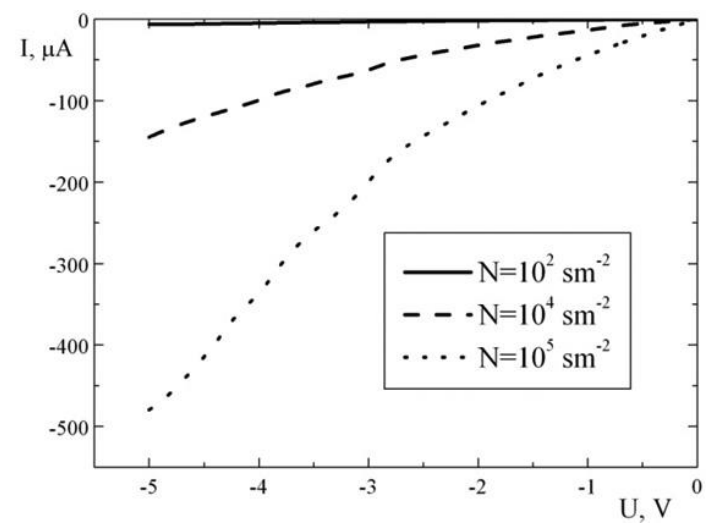

Fig. 1. Direct (a) and inverse (b) regions of VAC for the SBSs with different dislocation concentrations at their surfaces

a)

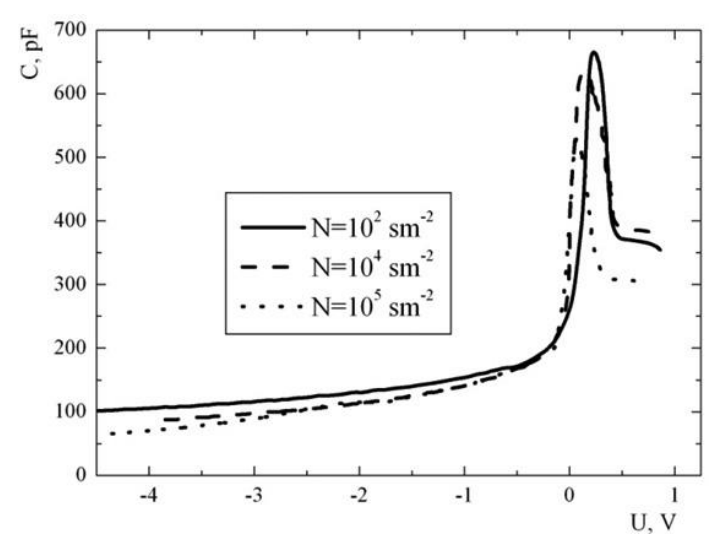

b)

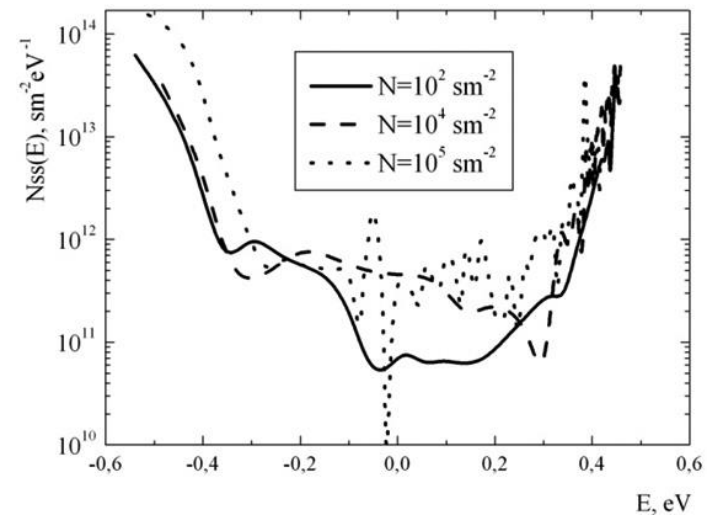

Fig. 2. CVC (a) and concentration distribution of the surface states inside the silicon bandgap (b) for the SBSs with different dislocation concentrations at their surfaces 
As shown by Slobodzyan et al. (2015), the maximum efficiency of generation of the surface states is peculiar for the defective near-surface layers where the mechanical strains are maximal. The source of these strains can be dislocations which contribute notably to the energy spectrum of the surface, thus changing the concentration distribution of the surface states inside the bandgap of silicon (see Fig. 2b). As a consequence, we have a change in the slope of the linear region of the CVC curve occurring at the positive voltages (see Fig. 2a).

Fig. 3 illustrates the radiation-stimulated changes typical for the direct region of VAC measured for the SBS created on the basis of silicon crystals. As seen from Fig. 3, the X-ray irradiation changes the slopes of this characteristic. Initially, we observe an increase in the direct current through the metal-semiconductor contact, though the doses of $390 \mathrm{~Gy}$ or higher cause decreasing VAC slope.

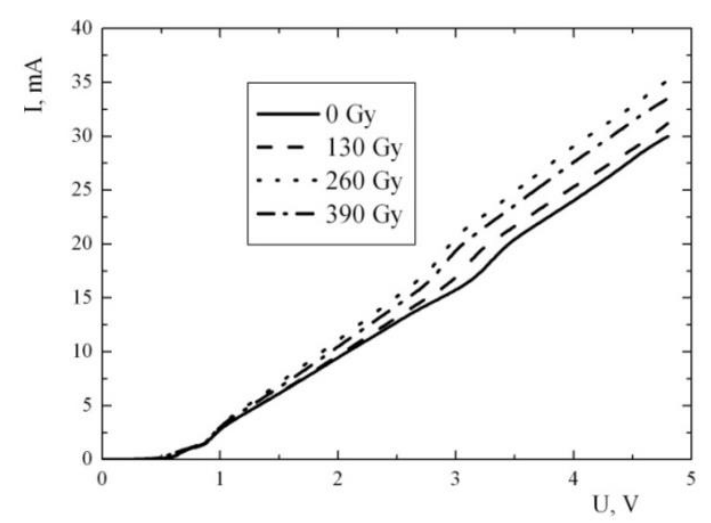

Fig. 3. Changes in the direct region of VAC for the SBS based on a dislocation-free silicon, which are induced by $X$-ray irradiation of different doses shown in the legend

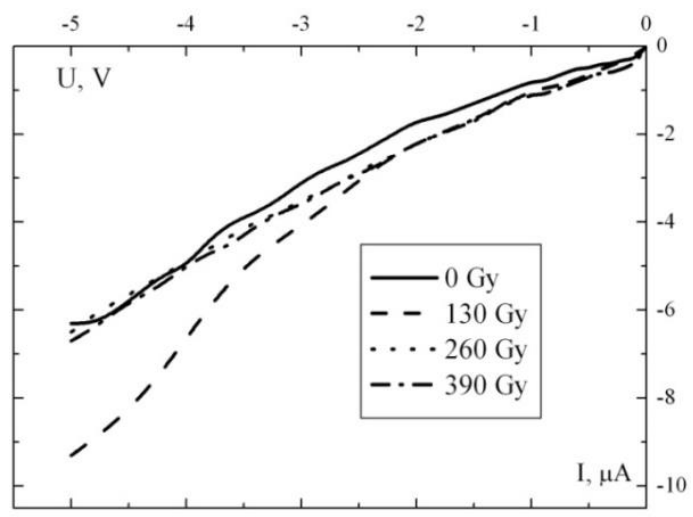

Fig. 4. Changes in the reverse region of VAC for the SBS based on a dislocation-free silicon, which are induced by X-ray irradiation of different doses shown in the legend

It is often assumed (Peka and Strikha, 1992) that the local areas are formed at the surface and in the near-surface layer of the crystal, which reveal higher contents of oxygen-vacancy and vacancy-impurity complexes. Some of these defects are in metastable states. This is because of differences among the charges localized at the vacancies and the impurities.

While analyzing the results obtained by us, it is necessary to consider the fact that generation of additional defects is not the only consequence of the irradiation. In addition, a process of radiation-stimulated 'ordering' of the defect structure takes place, which is known as 'the effect of small doses' (Pavlyk et al., 2012). On the initial stages of irradiating the SBS, there occurs partial reordering of the metastable near-surface defects. Then their charge state changes, together with the charge associated with the surface energy states. As a result of these changes, the concentration of charge carriers and the current associated with the direct VAC region increase. Further increase in the irradiation dose (>390 Gy) is accompanied by a domination of defectgeneration effect and so by gettering of the majority carriers at these defects. In its turn, this decreases the direct current.

As seen from Fig. 4, the radiation-stimulated changes occurring in the reverse region of the VAC are smaller. This fact can be explained by increasing probability of tunneling of the minority carriers through the barrier, with their subsequent localization at the imperfections of crystalline lattice.

As for the X-ray stimulated changes observed in the CVC of the SBS under study, Fig. 5a testifies that the increase in the absorbed dose (from 0 to $390 \mathrm{~Gy}$ ) imposes decreasing amplitude of a characteristic maximum typical of the CVC curve. Hence, the positive charge in the dielectric layer located between the metal and the semiconductor decreases. We notice that the mentioned layer could not be got rid of in the process of manufacturing the SBS.

a)

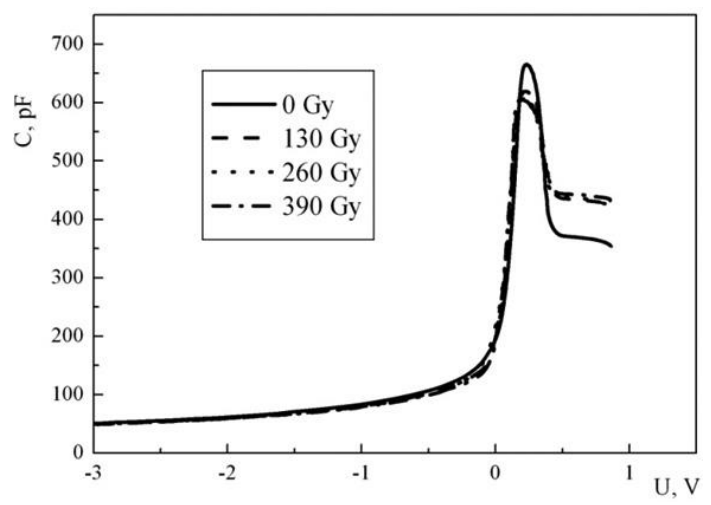

b)

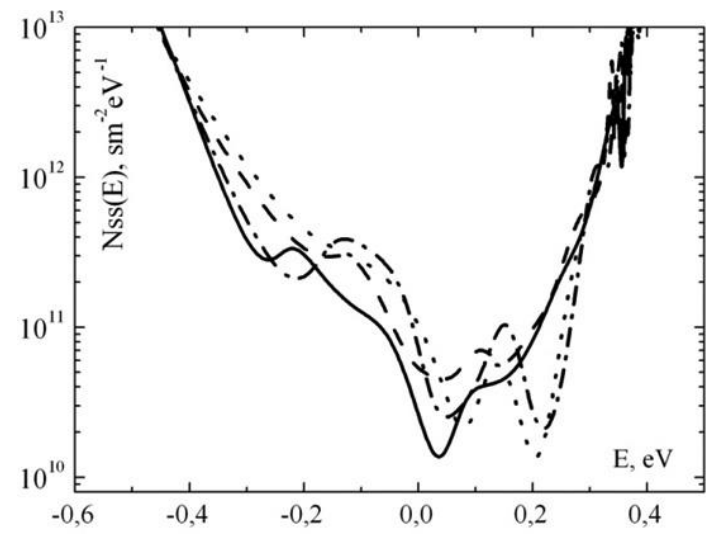

Fig. 5. Radiation-stimulated changes in CVC (a) and surface-state density (b) observed for the SBS based on dislocation-free silicon

Gradual capacity changes in the region of positive voltages occur whenever the absorbed dose increases. This reflects the changes in the charge of the space-charge region happening under the irradiation (i.e., a 'shelf' on the CVC). A 'critical' dose for the capacity dependence is $390 \mathrm{~Gy}$, since the capacity decreases under further increasing dose. These data correlate well with the 
radiation-stimulated changes seen in the VAC of our SBSs. They serve as a confirmation of reconstruction of the metastable defects, with the charge at these defects forming the region of the space charge.

It is known that the defects create additional discrete states at the surface of semiconductor crystals and contribute notably to the total spectrum of the surface-state density. Under $X$ irradiation, some reconstruction of the metastable defects can occur at the surface of the $p-S i: B$ structure $(\rho=240 \mathrm{Om} . \mathrm{cm})$. Moreover, additional defects are formed, of which occurrence depth only slightly differs from that of the former defects. Therefore the total spectrum of the surface states does not change in practice, though it shifts towards higher energies (see Fig. 5b).

Thus, the initial stages of irradiation ( $D<390 \mathrm{~Gy}$ ) of the SBSs based on dislocation-free silicon are governed by the mechanism of irradiation-induced ordering of the defect structure inside the near-surface silicon layers. The doses higher than $390 \mathrm{~Gy}$ change the prevailing mechanisms of interaction among the defects available in the structure and the radiation-stimulated defects.

The analysis of the radiation-stimulated changes in the VAC observed for the SBSs with different concentrations of dislocations suggests the following:

- A weak $(D<290 \mathrm{~Gy})$ irradiation treatment of our Si samples with the dislocation concentration of $N_{d}=10^{2} \mathrm{~cm}^{-2}$ leads to relatively small changes in both the direct and reverse VAC regions.

- The same treatment of the samples with $N_{d}=10^{4}-10^{5} \mathrm{~cm}^{-2}$ imposes a decrease in the direct current. The reason is increasing probability of scattering of carriers at the dislocations. Besides, the probability of gettering of the majority carriers at the dislocations increases with increasing coefficient of radiation-stimulated diffusion (Pavlyk et al., 2012).

- For the structures with $N_{d}=10^{4}-10^{5} \mathrm{~cm}^{-2}$, further increase in the absorbed dose up to $400 \mathrm{~Gy}$ is accompanied by increasing direct current, when compared with its initial value. The increase of the current may be explained by the effect of radiation-stimulated ordering of structural defects available in the crystal.

- Radiation-stimulated decrease in the reverse current, which is proportional to the density of the dislocations, is stipulated by generation of the minority carriers and their partial localization at the vacancies and dislocations.

That is why one can say that the defect state of the nearsurface layer of the silicon crystals where the SBSs are formed represents a crucial factor in the process of radiation-stimulated changes of their electro-physical characteristics (Pavlyk et al., 2013). The processes associated with the effect of $X$-rays are less important.

Figs. $6 \mathrm{a}$ and $6 \mathrm{~b}$ show the radiation-stimulated CVC changes observed for the SBSs with different dislocation concentrations at the crystal surface. Regardless of the quantity of dislocations under the barrier electrode, a peculiar feature of the radiationstimulated CVC changes is a decrease in the amplitude of a characteristic maximum observed in the region of positive voltages and, additionally, a lack of any changes in the density of surface states under the doses less than $390 \mathrm{~Gy}$. These features are the same as in the previous case of SBSs based on dislocationfree silicon. However, the capacity at the characteristic maximum begins to increase under the doses higher than $390 \mathrm{~Gy}$. As for the capacity corresponding to the space-charge region, it decreases at first and then starts to increase at the absorbed doses as low as $130 \mathrm{~Gy}$. The values of the characteristic doses for this structure correlate well with those causing the corresponding VAC changes.

a)

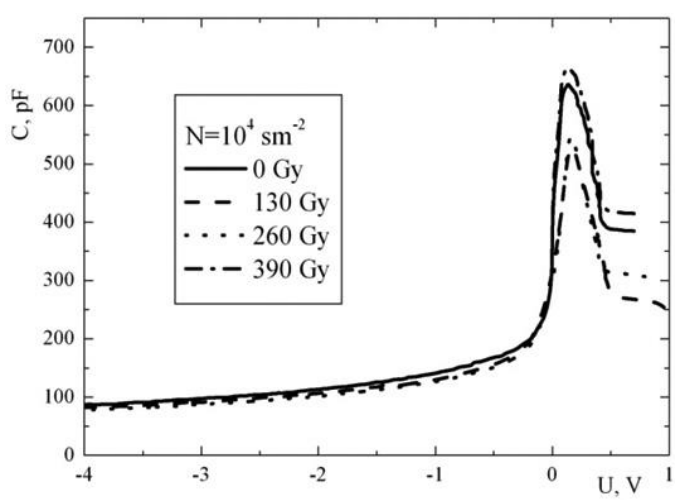

a')

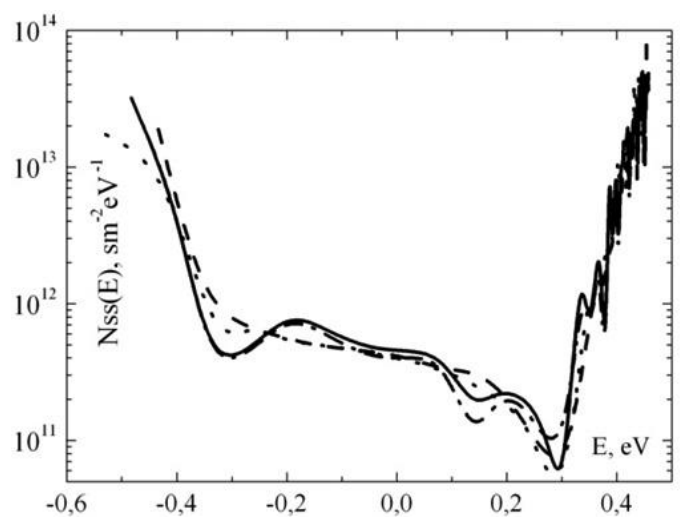

b)

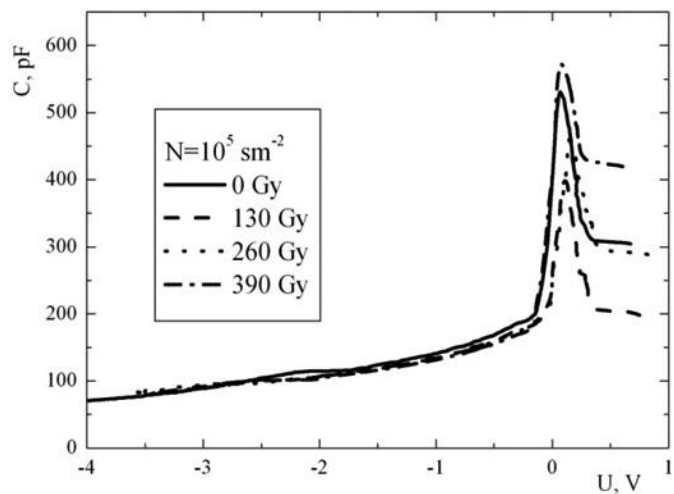

b')

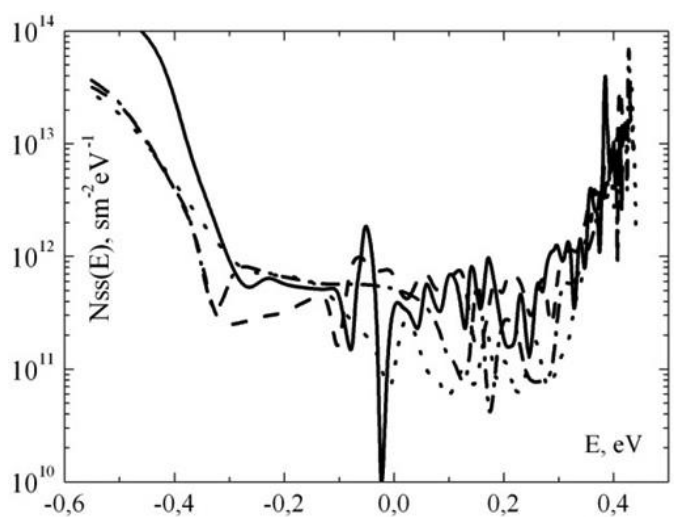

Fig. 6. Radiation-stimulated changes in CVC for the SBSs with different dislocation concentrations at the silicon surface $(a, b)$ and density distributions for the surface states inside the bandgap of silicon $\left(a^{\prime}, b^{\prime}\right)$ 
As seen from Fig. 6a', Fig. 6b', the dislocations contribute essentially to the energy spectrum of the surface states, of which charge changes after irradiation. It also accompanies the evolution of defect structure of the near-surface layers in the silicon crystals. Thus, we conclude that the increase in the density of the dislocations present in the near-surface crystal layer of p-Si stimulates efficient reconstruction of metastable and radiation-induced defects in the SBS.

The modeling of the underlying physical processes carried out by Pavlyk et al. (2013) has shown that the near-contact layers of $\mathrm{Al}$ and Si in the SBS are strained. Having performed similar calculations for the bismuth films, we arrive at considerably smaller mechanical tensions in the contact areas of the bismuth-silicon structures. This is a result of smaller differences between the corresponding lattice parameters and thermal expansion coefficients, as well as lower-temperature regime of Bi-film formation, when compared with Al films. In other words, the thickness of the strained layers is an order of magnitude smaller, being about one or two atomic layers in the contact region of silicon. Then the depth of capture of the defects from the crystal bulk is also smaller for this region. The results of our modeling are shown in Fig. 7 .

a)

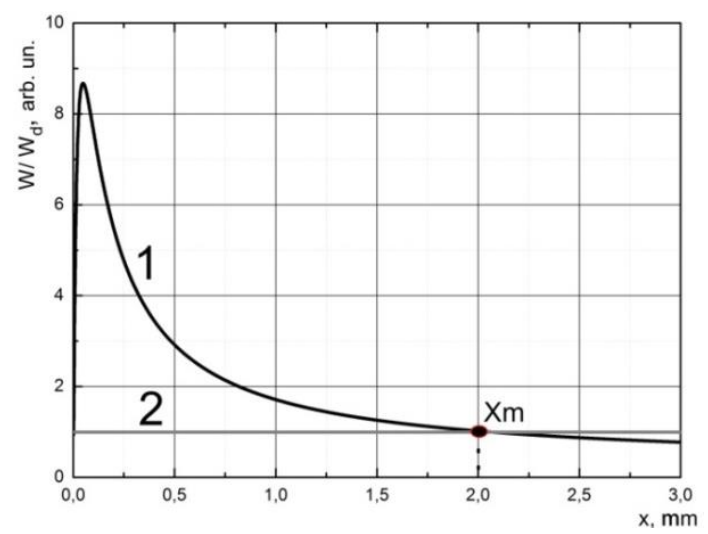

b)

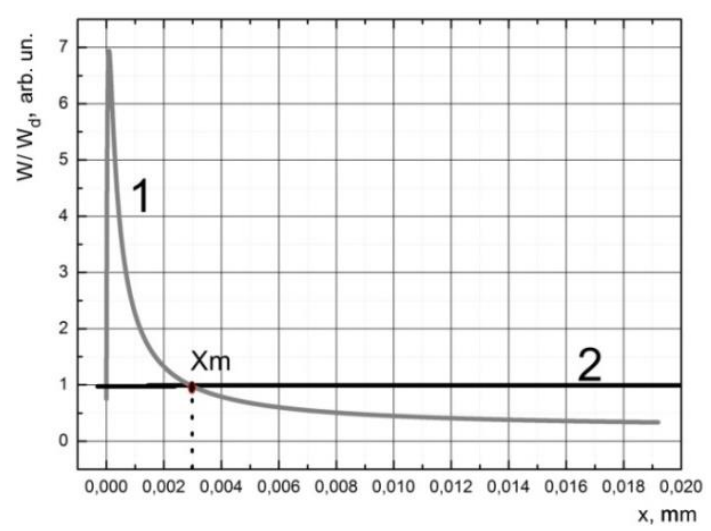

Fig. 7. Dependences of total energy of the strained $\mathrm{Al}-\mathrm{Si}(\mathrm{a})$ and $\mathrm{Si}-\mathrm{Bi}$ (b) layers (curve 1) and dislocation energy (curve 2) on the distance from the crystal surface: $\mathrm{Xm}$ is the maximal depth of defect capture from the crystal volume

The studies of the surface structure performed after chemical etching of the metal films and further selective etching have shown that, in practice, there is no defect layer under the bismuth films. Since the dislocations impose mechanical tension fields in their vicinities, they represent efficient gettering centers for different types of point defects and impurities in the crystal. Application of external mechanical field or temperature treatment of the samples stimulate a motion of dislocations, which migrate through the crystal lattice (e.g., the loop-shaped dislocations with fixed ends can become curved) and capture defects from the crystal bulk. The dislocations move inside the near-surface layer subjected to mechanical tensions, which are caused by a difference in the lattice parameters of silicon and the aluminum film. There they leave the defects localized around the dislocation cores. After the tension is unloaded, the dislocation is becoming 'straightened' and turns back to its initial state. The defects captured remain to be in the deformation-potential field of the near-surface layer.

It is important that the defect-type near-surface layer is formed only under the aluminum films at high enough temperatures, and precipitated aluminum is not saturated by silicon up to its maximal solubility of $1.6 \%$. Then one can assume a low-temperature diffusion of the silicon atoms into the polycrystalline Al film. This assumption is confirmed by the fact that, during the formation of SBSs, a significant amount of defects (e.g., dislocations, grain boundaries, etc.) is formed in the bulk of the film. These defects act as pathways of accelerated diffusion. Moreover, it is necessary to take into consideration that the mechanical tensions in the contact area weaken interactions of silicon atoms located in the crystalline lattice sites. As a result of all of these processes, a layer is formed in the region of silicon nearby the boundary, which is enriched with vacancies, interstitial silicon atoms and the other impurity atoms.

\section{CONCLUSIONS}

1. As a result of mechanical tensions present in the strained defect layer, the defects are captured from the crystal bulk, thus leading to the changes observed in electro-physical characteristics of the SBS samples.

2. The irradiation triggers the two competing processes: the generation of additional defects and the change in charge state of the existing defects. Depending on the defect structure of the silicon crystals, one of these mechanisms can prevail on a particular stage of irradiation.

3. Dislocations act as getters for charge carriers, thus resulting in significant differences observed for the characteristics of electronic devices made on the bases of dislocation-including and dislocation-free silicon crystals.

\section{REFERENCES}

1. Borkovska O.Y, Dmitruk P.L., Litovchenko V.H. (1984), Effect of radiation ordering in heterojunctions of (n-Si)-(p-GaP), Semiconductors, 18(10), 1808-1810.

2. Mahkamov S., Tursunov N.A., Ashurov M. (1999), About the peculiarities of formation of radiation defects in silicon structures, Technical Physics, 69(1), 121-123.

3. Nikolaev D.V., Antonova I.V., Naumova O.V. (2003), Charge accumulation in oxide and interface states of silicon-on-insulator structures after irradiation by electrons and $y$-rays, Semiconductors, 37(4), 443-449.

4. Marchenko I.G., Zhdanovich N.E. (2010), Influence of irradiation by electrons on the electrical parameters of silicon $p$-n-structures, weakened by aluminum screens, Technical Physics, 36(10), 45-51.

5. Kalinina E.V., Kossov V.G., Yafaev R. R. (2010), High-temperature radiation-strong rectifier based on $4 \mathrm{H}-\mathrm{SiC}$ alumimium ion implanted $p^{+}$-n-junctions, Semiconductors, 44(6), 807-815. 
6. Dolgolenko A.P., Litovchenko P.G., Varentsov M.D. (2006), Particularities of the for-mation of radiation defects in silicon with low and high concentration of oxygen, Physica Status Solidi, 243(8), 1842-1852.

7. Makara V.A., Vasiliev M.A., Steblenko L.P. (2008), Caused by magnetic field changes of impurity composition and microhardness of silicon crystals, Semiconductors, 42(9), 1061-1064.

8. Skvortsov A.A., Orlov A.M., Solov'ev A.A. (2009), Magnetoplastic effect in silicon: the search for new methods of management of structure-sensitive properties of elemental semiconductors, Physics of the Solid State, 51(12), 2304-2308.

9. Mudriy S.I., Kulyk Y.O., Steblenko L.P. (2010), Change of internal stress and lattice parameter of silicon crystals, stimulated by the combined influence of $\mathrm{X}$-ray irradiation and a magnetic field, Physics and Chemistry of Solid State, 11(2), 334-337.

10. Slobodzyan D.P., Pavlyk B.V., Kushlyk M.O. (2015), Features of influence of $x$-radiation and magnetic field on the electrical characteristics of barrier structures based on $p$-si with dislocation designed for solar energy, J. Nano- Electron. Phys., 7(4), 04051-1 04051-5.

11. Peka G.P., Strikha V.I. (1992), Surface and contact phenomena in semiconductors, Kyiv, Lybid.

12. Pavlyk B.V., Slobodzyan D.P., Kushlyk M.O. (2012), Quality of the $p$-Si crystal surface and radiation-stimulated changes in the characteristics of Bi-Si-Al surface-barrier structures, Semiconductors, 46(8), 1017-1021.

13. Pavlyk B.V., Slobodzyan D.P., Kushlyk M.O. (2013), Electrophysical characteristics of near-surface layers in $p$-si crystals with sputtered al films and subjected to elastic deformation, Ukr. J. Phys., 58(8), 742-747. 